\title{
Des corps flottants aux miroirs déformants
}

\section{Une cité de la géométrie à l'Université de Nice Sophia Antipolis}

Pierre Coullet $^{(1)}$ (pierre.coullet@unice.fr) et Jean-Luc Filippi ${ }^{(2)}$ (jean-luc.filippi@unice.fr)

(1) INLN, UMR 7335 CNRS-Université de Nice Sophia Antipolis, 06560 Valbonne.

(2) Institut Culture Science Alhazen, Université de Nice Sophia Antipolis, Campus Valrose, B.P. 2135, 06103 Nice Cedex 2.

Dans cet article, nous examinons deux problèmes classiques de bifurcation : I'instabilité d'un corps de révolution flottant étudiée par Archimède il y a 2300 ans, et la multiplicité des images observées dans les miroirs déformants, un problème résolu par Alhazen au $11^{\mathrm{e}}$ siècle.

Nous avons choisi ces deux exemples concrets afin d'illustrer l'esprit de la Cité de la Géométrie que nous avons mise en place à I'Université de Nice Sophia Antipolis. Une cinquantaine d'expériences de statique, d'optique et de mécanique permettent de retracer l'histoire des idées qui, d'Euclide à Newton, ont permis l'émergence de la physique moderne.

\section{Présentation}

L'architecture navale et la stabilité des vaisseaux furent un des thèmes majeurs de l'activité de Pierre Bouguer, dont nous publions une biographie dans ce numéro de Reflets de la physique. Il nous a semblé intéressant d'y associer la contribution de Pierre Coullet, qui intéressera les physiciens à plusieurs titres.

L'étude des bifurcations auxquelles ces analyses de stabilité donnent accès (P. Coullet a reçu le prix Holweck pour ses travaux sur ce thème) permet de mettre en regard en particulier des problèmes de mécanique (bifurcations et chaos), et de caustiques en optique.

Ces études de stabilité de coques mettent en jeu des contributions sur une large période d'histoire des sciences, d'Archimède aux travaux de Bouguer, Newton et Euler, à partir d'une approche géométrique de la Science, souvent négligée aujourd'hui. Pierre Coullet s'est intéressé aux contributions de la science arabe à la géométrie, l'optique et l'astronomie ; l'Institut Culture Science de l'Université de Nice Sophia Antipolis porte d'ailleurs le nom d'Alhazen, le grand opticien et géomètre arabe qui vécut vers l'an 1000. Dans le cadre d'un programme d'actions culturelles à Nice, Pierre Coullet a mis en place un ambitieux projet : la "Cité de la Géométrie ». Au long d'une promenade scientifique dans un espace du "Château » du campus Valrose, les visiteurs, adultes et collégiens, pourront voir et faire de superbes expériences de mécanique (stabilité et chaos), d’optique géométrique et, dans le même temps, suivre la progression des idées scientifiques sur ces thèmes.

Étienne Guyon

\section{Vingt siècles de représentation géométrique du monde}

Pendant près de vingt siècles, d'Euclide à Newton, la modélisation des phénomènes naturels se fit exclusivement avec les méthodes de la géométrie élémentaire. C'est à partir du cercle que l'on construit le cône et c'est à partir du cône que se définissent les coniques. Ces courbes remarquables du second degré que sont l'ellipse, la parabole et l'hyperbole, initialement introduites pour résoudre des équations de degré inférieur ou égal à quatre, devaient apparaître de façon étonnante comme les modèles mathématiques de phénomènes naturels. Les miroirs et les lentilles " ardentes ", qui concentrent en un point unique les rayons issus d'une source ponctuelle de lumière, sont des coniques. Le fameux problème de deux corps en interaction gravitationnelle, résolu par Newton, admet comme solution générale des orbites elliptiques ou hyperboliques et plus singulièrement circulaires et paraboliques. L'étude des coniques fut poussée très loin par Apollonius ${ }^{(a)}$, dont la renommée lui valut le nom de "grand géomètre ". 


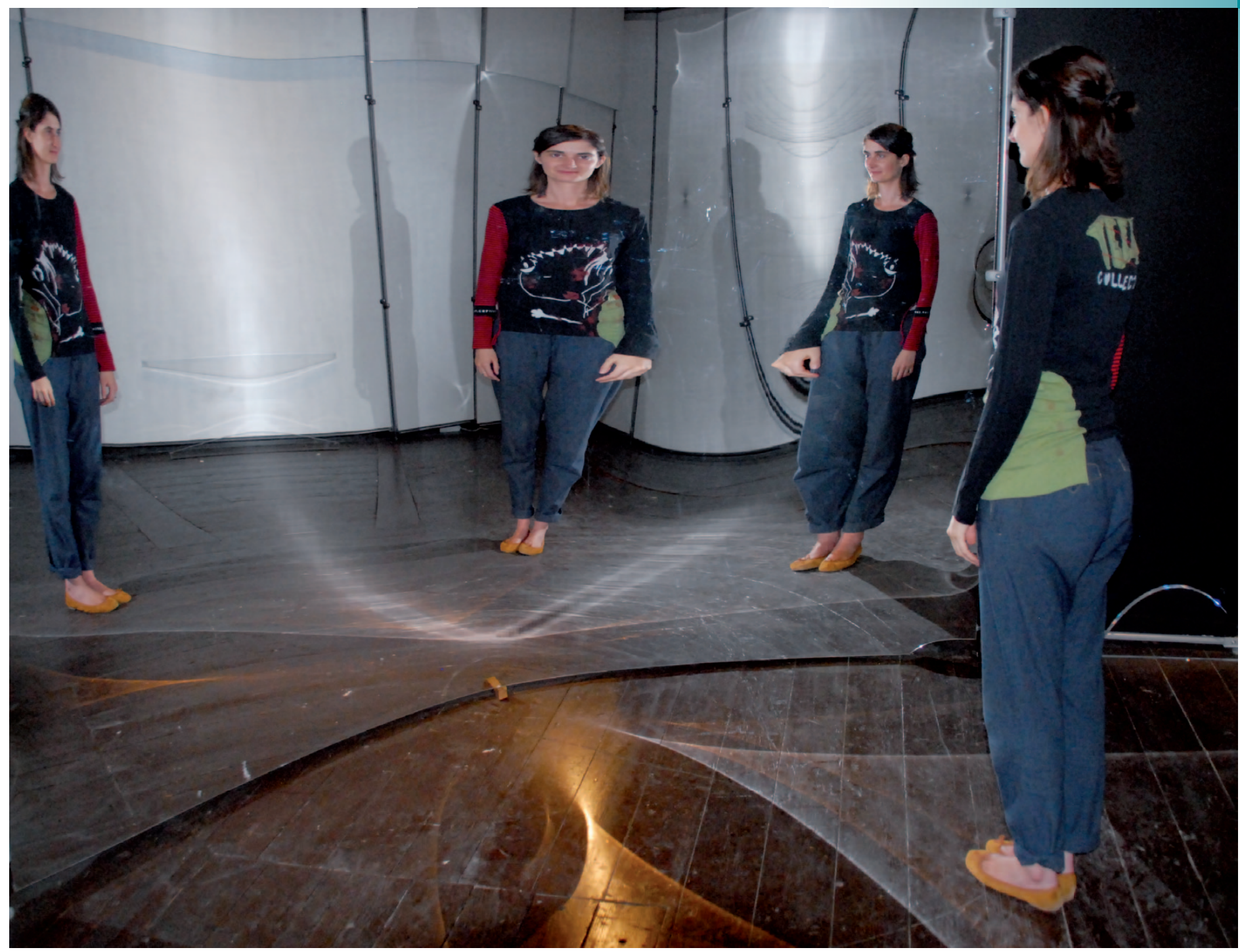

Image triple dans un miroir cylindrique de section parabolique (voir texte p. 37). La personne photographiée est Laurie Chiara, la journaliste scientifique de l'Institut Culture Science Alhazen. La courbe caustique blanche est l'enveloppe des rayons lumineux issus du flash du photographe. La position de Laurie à l'intérieur de cette courbe permet d'interpréter l'existence des trois images. Remarquons également que l'image centrale est inversée gauche-droite par rapport aux deux autres. La relation entre les images observées sur le miroir et le problème d'Alhazen est assez subtile, puisqu'il s'agit en effet d'une source étendue $A(x, y, z)$, où $x, y$ et $z$ représentent des coordonnées du plan de la pellicule de l'appareil de photo que l'on observe au travers du diaphragme B de l'appareil que l'on peut considérer comme ponctuel. II y a donc autant de problèmes d'Alhazen à résoudre que de points sources $A(x, y, z)$. Cependant, la position de Laurie à l'intérieur de la courbe caustique permet de conclure à l'existence de trois solutions pour chaque point de la source. La direction z n'est en principe pas pertinente puisque le miroir n'est pas courbé dans cette direction.

\section{Les lignes extrémales d'Apollonius}

Dans son cinquième livre, Apollonius étudie un problème abstrait d'extrema qui devait avoir des applications physiques importantes. Il se propose de trouver, pour une courbe conique donnée et un point A quelconque, les extrema des distances des segments joignant ce point à la courbe. Ces « lignes extrémales » sont normales à la courbe, c'est-à-dire orthogonales à sa tangente.

Illustrons les résultats d'Apollonius dans le cas de la parabole. Selon la position de A, il montre l'existence de un ou trois extrema (fig. 1). Le changement du
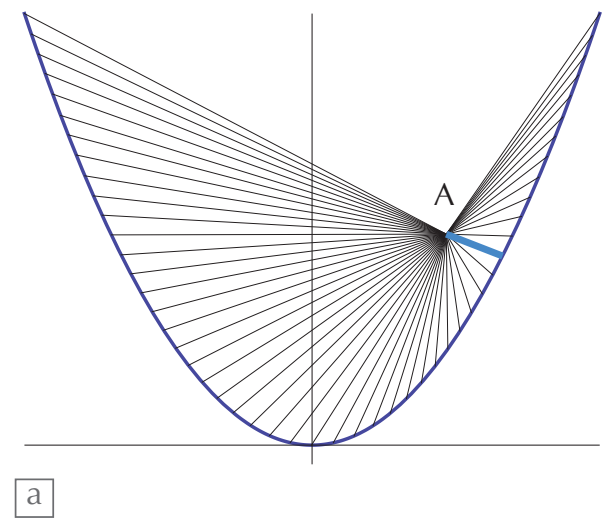

1. Les lignes extrémales. (a) Lorsque le point A est « proche » de la parabole, une seule ligne extrémale existe et c'est un minimum. (b) Lorsque A est plus éloigné, il existe trois extrema, deux minima et un maximum. Les minima sont représentés en bleu et le maximum en noir. 
$>>$

nombre d'extrema survient sur une courbe singulière, l'enveloppe de la famille de ses normales notée $\mathrm{H}$ sur la figure 2. Huygens, dans sa théorie des développées, retrouvera cette courbe et la nommera parabole semi-cubique. Elle est de la forme $\mathrm{y}^{3}=\mathrm{x}^{2}$. $\mathrm{H}$ est la développée de la parabole, c'està-dire le lieu de ses centres de courbure.

Lorsque $A$ est sur l'axe de la parabole, s'il est situé en dessous du minimum $M$ de l'enveloppe des normales, il n'existe alors qu'une ligne extrémale. C'est un minimum. Au-dessus de $M$, il existe trois lignes extrémales. L'une, maximale, est sur l'axe et les deux autres, symétriques de part et d'autre de l'axe, sont minimales. On reconnaît alors la fameuse bifurcation " fourche " tant étudiée dans les dernières décennies. Lorsque $A$ n'est pas sur l'axe de la parabole, on doit distinguer les cas où il se situe au-dessus ou en dessous de l'enveloppe des normales. Dans le premier cas, trois extrema existent et la situation est qualitativement la même que dans le cas symétrique, la ligne maximale est comprise entre les deux lignes minimales. Dans le second cas, il n'existe qu'un extremum et c'est un minimum.

La question des extrema est devenue centrale dans la formulation variationnelle des lois de la physique. Les équilibres sont des extrema de l'énergie potentielle, et le trajet des rayons lumineux entre deux points est un extremum du chemin optique.

Nous considérons ici deux problèmes qui nous ramènent à l'analyse d'Apollonius.

\section{La stabilité des corps flottants, Archimède, Livre II}

Dans le Livre I des corps flottants, Archimède introduit sa fameuse loi. Dans le deuxième livre, il étudie la stabilité des équilibres d'un corps flottant possédant une symétrie de révolution et il montre, en particulier, dans le cas d'un paraboloïde de révolution plein que l'équilibre vertical peut être rompu spontanément. Il introduit deux paramètres sans dimension, le rapport d'aspect $\eta=H / R$ du paraboloïde où $H$ représente sa hauteur mesurée à partir de son sommet $S$ et $R$ le rayon du disque de la face plane supérieure qu'il nomme la base, et $\rho=d_{f} / d_{s}$ le rapport des densités du fluide et du paraboloïde qu'il considère comme homogène. Il détermine en fonction de ces paramètres la stabilité de l'équilibre vertical et calcule l'angle d'inclinaison du corps lorsqu'il devient instable. Il s'agit là du premier calcul exact de bifurcation fourche. Regardons ce qu'en dit Lagrange en 1778 dans sa Mécanique Analytique.

«Tels sont les objets du premier livre. Dans le second, Archimède donne d'après les mêmes principes, les lois de l'équilibre de différents solides formés par la révolution des Sections coniques, et plongés dans des fluides plus pesants que ces corps ; il examine les cas où ces conoïdes peuvent $y$ demeurer inclinés, ceux où ils doivent s'y tenir debout, et ceux où ils doivent culbuter ou se redresser. Ce Livre est un des plus beaux monuments du génie d'Archimède, et renferme une théorie de la stabilité des corps flottants à laquelle les modernes ont peu ajouté. "

Archimède se limite, pour des raisons techniques, au cas où la ligne de flottaison ne touche pas la base de la parabole. Dans la présentation faite ici, par souci de simplicité, nous avons remplacé le paraboloïde par un cylindre infini de section parabolique et introduisons une idée de Bouguer et Euler, généralisée magistralement par Dupin. Elle permet de ramener ce problème à la recherche des lignes extrémales d'Apollonius. À la manière de Bouguer et Euler, c'est la ligne de flottaison (fig. 3) que l'on inclinera plutôt que le navire. L'égalité des surfaces du secteur parabolique limité par la ligne de flottaison et la coque est une condition nécessaire d'équilibre, puisque l'on suppose que la poussée d'Archimède demeure constante lors de l'inclinaison du navire. Notons $-r$ et $r$, où $r=R \rho^{1 / 3}$, les abscisses des extrémités de la ligne de flottaison lorsque le navire est en position verticale. Lors de son basculement, la partie immergée du navire conserve le même volume.

Lorsque le navire s'incline d'un angle $\theta$, les abscisses des extrémités des lignes de flottaison, calculées pour maintenir l'équilibre, sont simplement données par $(-r+\mathrm{e}, r+\mathrm{e})$, où $-R+r<\mathrm{e}<R-r$ (restriction similaire à celle d'Archimède), $R$ est la demi-largeur de la base $R \_R_{+}$, et $\mathrm{e}=\left(R^{2} / 2 H\right) \tan (\theta)$. Le centre de poussée d'Archimède du navire en position verticale est situé sur l'axe de la parabole à la

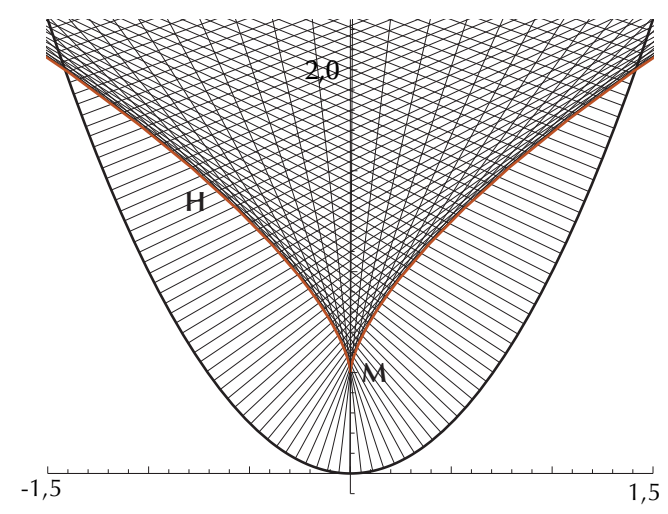

2. Les normales de la parabole et leur enveloppe. La courbe rouge représente l'enveloppe des normales. Son minimum est noté $M$. Au-dessus de cette courbe, en chaque point passent trois normales.

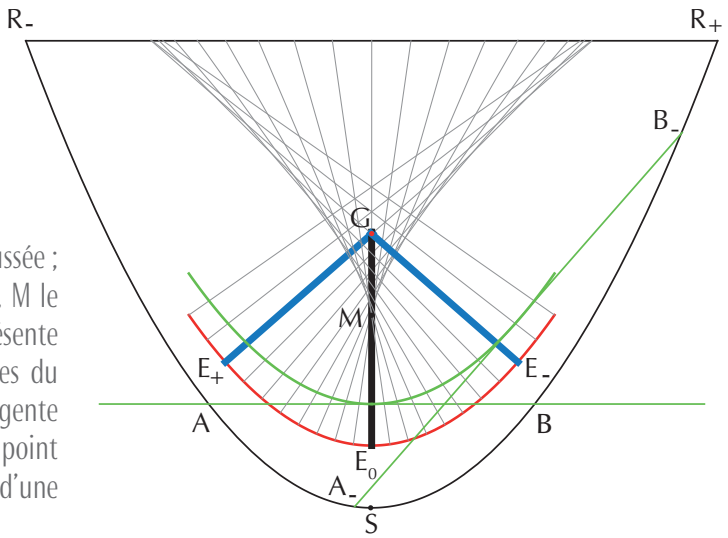

3. La construction de Bouguer : en rouge, la ligne des centres de poussée en vert, l'enveloppe des lignes de flottaison. G est le centre de gravité, M le métacentre de Bouguer. Les équilibres sont notés $E_{+}, E_{-}$et $E_{0}$. $E_{0}$ représente l'équilibre instable vertical, $E_{+}$et $E_{-}$représentent les équilibres stables $d u$ navire incliné. La ligne de flottaison $A_{-} B_{-}$est orthogonale à GE_ et tangente à son enveloppe. Au-dessus de l'enveloppe des normales, par chaque point passent trois normales, par-dessous une seule. L'enveloppe est le lieu d'une bifurcation où deux normales apparaissent ou disparaissent. . 


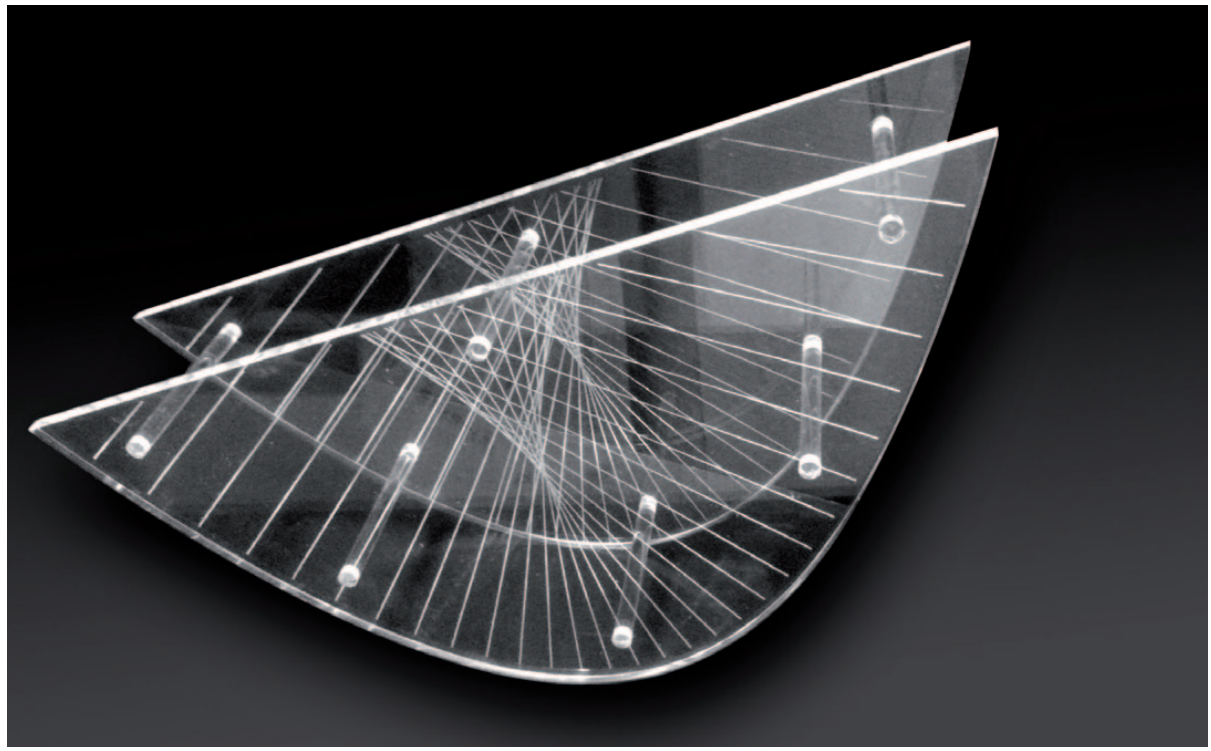

4. Un culbuto parabolique. C'est la limite $\rho=0$ du problème d'Archimède. Dans ce cas, la poussée d'Archimède devient simplement la réaction du support. La ligne de flottaison sur lequel il roule est la surface plane sur laquelle il est posé.

hauteur $3 h / 5$, et noté $\mathrm{E}_{0}$ sur la figure 3, où $h=H \rho^{2 / 3}$. Lorsque la ligne de flottaison s'incline, le centre de poussée, centre de masse de la partie immergée du navire, décrit une parabole de même profil que celui du navire mais décalée vers le haut de $3 h / 5$. On nommera ligne des centres de poussée cette courbe, représentée en rouge sur la figure 3. Dupin montre alors que tout se passe comme si le navire se comportait comme un "culbuto " de section parabolique (fig. 4), dont le profil est la ligne des centres de poussée, qui roulerait sur la ligne de flottaison passant par $\mathrm{E}_{0}$. L'enveloppe des normales de ce culbuto fictif est la fameuse courbe cuspoïdale (parabole semi-cubique, de la forme $\mathrm{y}^{3}=\mathrm{x}^{2}$ ) d'Apollonius-Huygens. Avec Bouguer, nous appellerons "Métacentre " le point de rebroussement ( $c u s p)$ de cette courbe. C'est le centre de courbure du sommet du culbuto fictif. Si le centre de gravité du navire, situé sur son axe à la hauteur $3 H / 5$ se situe en dessous du métacentre, son seul équilibre sera en position verticale alors qu'il s'inclinera vers l'une des deux positions stables (minimisant la distance, donc l'énergie potentielle) s'il se situe au-dessus. On voit ainsi que la recherche des équilibres, même dans le cas où la distribution des masses dans le navire ne serait pas homogène, revient à trouver les distances extrémales du centre de gravité à la courbe de poussée. tracé en vert sur la figure 3 l'enveloppe des lignes de flottaison. Il s'agit également d'une parabole de même profil que celle du navire, mais décalée vers le haut de $h$. Elle permet de construire graphiquement la ligne de flottaison pour un point quelconque de la courbe des centres de poussée.

\section{Le problème d'Alhazen du miroir parabolique}

On considère dans le plan un miroir parabolique et deux points $A$ et $B$ situés dans la partie concave de la parabole. La question posée par Alhazen est la suivante : combien de rayons lumineux issus de $A$ passent par $B$ après une réflexion sur le miroir ? Le cas simple où $A$ et $B$ sont confondus se ramène directement au problème d'Apollonius, puisqu'alors il s'agit simplement de chercher les extrema de la distance d'un point à la parabole, conséquence immédiate du principe de Fermat. Les effets des bifurcations observées lorsque l'on change la position du point se manifestent clairement lorsque l'on se regarde dans un miroir parabolique de taille importante. On observe une ou trois images selon sa position. Elles sont inversées " droite-gauche » ou non selon que la distance au miroir est minimale ou maximale (dans le cas où $A$ et $B$ sont distincts, voir la photo page 35 ).
Pour compléter l'analyse, nous avons

\section{Notes et Références}

(a) Apollonius de Perge (vers 262 - 190 av. J.-C.), géomètre et astronome grec de l'école d'Alexandrie. Son œurre fondamentale est Les coniques, composée de huit volumes.

1 www.ics-unice.fr/category/la-cite-de-la-geometrie-2/

2• www.ac-nice.fr/plpms/site/index.php?option=com content\&view=article\&id=46:les-cites-de-lageometrie\&catid=63:didactique- $-\mathrm{a}$ pedagogie\&ltemid=36

3•P. Coullet, "Bifurcations at the Dawn of Modern Science", www.ics-unice.fr/wp-content/ uploads/2012/10/Bifurcation.pdf

\section{La Cité de la Géométrie}

Nous avons choisi ces deux exemples concrets afin d'illustrer l'esprit de la Cité de la Géométrie que nous avons mise en place à l'Université de Nice Sophia Antipolis [1]. Une cinquantaine d'expériences de statique, d'optique et de mécanique permettent de retracer l'histoire des idées qui, d'Euclide à Newton, ont permis l'émergence de la physique moderne. Assorties d'explications et de simulations interactives, elles sont dédiées à un enseignement original des sciences, où l'histoire est revisitée à la lumière de la science contemporaine. Ce projet se développe aujourd'hui, en partenariat avec le rectorat [2] et le conseil général des AlpesMaritimes, en direction de huit collèges du département où de grands miroirs ont été installés.

\section{Remerciements}

Pierre Coullet tient à remercier Albert Marouani, président honoraire de l'Université de Nice Sophia Antipolis, pour avoir permis la réalisation de ce projet, Étienne Guyon pour avoir suggéré l'écriture de cet article et l'intérêt qu'il a manifesté pour ce travail, Jean-Pierre Hulin pour ses critiques constructives et les nombreuses corrections qu'il a apporté à ce texte, Yves Pomeau pour de nombreuses discussions, et Roshdi Rashed pour ses traductions et commentaires des travaux d'Apollonius, notamment du Livre V, et la patience et la gentillesse dont il a fait preuve pour répondre à toutes mes questions. Finalement, P.C. remercie le CNRS qui lui a permis de reprendre une activité scientifique, après de (trop) nombreuses années de vice-présidence universitaire.

Le lecteur intéressé trouvera plus de détails dans un article sous presse aux Comptes Rendus de l'Académie des sciences, Section Mécanique [3]. 